\title{
Smad4 haploinsufficiency: a matter of dosage
}

Paola Alberici ${ }^{1}, 7$, Claudia Gaspar ${ }^{1}$, Patrick Franken ${ }^{1}$, Marcin M Gorski 2,8 , Ingrid de Vries ${ }^{1}$, Rodney J Scott ${ }^{3}$, Ari Ristimäki ${ }^{4,6}$, Lauri A Aaltonen ${ }^{5,6}$ and Riccardo Fodde*1

Address: ${ }^{1}$ Department of Pathology, Josephine Nefkens Institute, Erasmus MC, Rotterdam, The Netherlands, ${ }^{2}$ Department of Biochemistry, Erasmus MC, Rotterdam, The Netherlands, ${ }^{3}$ Newcastle Bowel Cancer Research Collaborative, Hunter Medical Research Institute, John Hunter Hospital and The University of Newcastle, Newcastle, Australia, ${ }^{4}$ Division of Pathology HUSLAB and Haartman Institute, Helsinki University Central Hospital, Helsinki, Finland, ${ }^{5}$ Department of Medical Genetics, HUSLAB and Haartman Institute, Helsinki University Central Hospital, Finland, ${ }^{6}$ Genome Scale Biology Program, Biomedicum Helsinki, University of Helsinki, Helsinki, Finland, ${ }^{7}$ Current address: IFOM -The FIRC Institute of Molecular Oncology, IFOM-IEO Campus, Milano, Italy and ${ }^{8}$ Current address: Department of Experimental Oncology, European Institute of Oncology (IEO), IFOM-IEO Campus, Milano, Italy

Email: Paola Alberici - paola.alberici@ifom-ieo-campus.it; Claudia Gaspar - c.gaspar@erasmusmc.nl;

Patrick Franken - p.f.franken@erasmusmc.nl; Marcin M Gorski - marcin.gorski@ifom-ieo-campus.it; Ingrid de Vries - i.devries.1@erasmusmc.nl; Rodney J Scott - rodney.scott@newcastle.edu.au; Ari Ristimäki - ari.ristimaki@helsinki.fi; Lauri A Aaltonen - Lauri.Aaltonen@Helsinki.Fi; Riccardo Fodde* - r.fodde@erasmusmc.nl

* Corresponding author

Published: 3 November 2008

PathoGenetics 2008, I:2 doi:10.1186/1755-8417-1-2
Received: 3 July 2008

Accepted: 3 November 2008

This article is available from: http://www.pathogenetics.com/content/I/I/2

(c) 2008 Alberici et al; licensee BioMed Central Ltd.

This is an Open Access article distributed under the terms of the Creative Commons Attribution License (http://creativecommons.org/licenses/by/2.0), which permits unrestricted use, distribution, and reproduction in any medium, provided the original work is properly cited.

\begin{abstract}
Background: The inactivation of tumor suppressor genes follows Alfred Knudson's 'two-hit' model: both alleles need to be inactivated by independent mutation events to trigger tumor formation. However, in a minority of tumor suppressor genes a single hit is sufficient to initiate tumorigenesis notwithstanding the presence of the wild-type allele, a condition known as haploinsufficiency. The SMAD4 gene is an intracellular mediator of the TGF- $\beta$ and BMP signal transduction pathways and a tumor suppressor involved in pancreatic and colorectal tumorigenesis. In Smad4-mutant mouse models, haploinsufficiency characterizes the development of gastrointestinal polyps with initial retention of the wild-type allele and protein expression within the nascent tumors and in their direct microenvironment. Similarly, germline SMAD4 mutations are responsible for a subset of patients affected by juvenile polyposis syndrome, an autosomal dominant intestinal cancer syndrome. To date, the molecular and cellular consequences of SMAD4 haploinsufficiency on TGF- $\beta$ and BMP signaling and on genome-wide gene expression have not been investigated.
\end{abstract}

Results: Here we show that, similar to previous observations in Smad4-mutant mouse models, haploinsufficiency characterizes a substantial fraction of the juvenile polyps arising in patients with germline SMAD4 mutations. Also, mouse embryonic and intestinal cells heterozygous for a targeted Smad4 null mutation are characterized by a corresponding 50\% reduction of the Smad4 protein levels. Reporter assays revealed that mouse Smad $4^{+/}$- cells exert intermediate inhibitory effects on both TGF- $\beta$ and BMP signaling. Genome-wide expression profiling analysis of Smad4+/- and Smad4I- cells pinpointed a subset of dosage-dependent transcriptional target genes encompassing, among others, members of the TGF- $\beta$ and Wnt signaling pathways. These SMAD4 dosage-dependent 
transcriptional changes were confirmed and validated in a subset of target genes in intestinal tissues from juvenile polyposis syndrome patients.

Conclusion: Smad4 haploinsufficiency is sufficient to significantly inhibit both TGF- $\beta$ and BMP signal transduction and results in the differential expression of a broad subset of target genes likely to underlie tumor formation both from the mesenchymal and epithelial compartments. The results of our study, performed in normal rather than tumor cells where additional (epi-) genetic alterations may confound the analysis, are relevant for our understanding and elucidation of the initial steps underlying SMAD4-driven intestinal tumorigenesis.

\section{Background}

Haploinsufficiency is defined as the condition where mutation or loss of a single allele is sufficient to alter the phenotype of a diploid cell [1]. Haploinsufficiency at a tumor suppressor locus may overcome the need for somatic loss or mutation of its wild-type allele, predicted as the rate-limiting event for tumor development by the Knudson's 'two-hit' model [2]. To date, experimental evidence for haploinsufficiency in cancer predispositions comes from the analysis of tumors obtained from mouse models or hereditary cancer patients carrying heterozygous null mutations at known tumor suppressor genes [3]. The absence of the second hit in a subset of these tumors has been attributed to many causes, including inactivation of the remaining allele by alternative mechanisms such as epigenetic silencing, mutations in non-coding sequences, or to limited sensitivity of the employed mutation detection protocol. However, bona fide haploinsufficiency has been demonstrated for a subset of tumor suppressor loci including SMAD4 [4-6], an intracellular mediator of the TGF- $\beta$ and BMP signal transduction pathways $[7,8]$. Upon TGF- $\beta$ or BMP signaling, SMAD4 binds to the receptor-activated SMADs and translocates to the nucleus where it modulates the transcription of a broad spectrum of target genes involved in cell growth inhibition, apoptosis, differentiation, and matrix production [79]. Somatic SMAD4 gene mutations are found in only a fraction of advanced sporadic colorectal cancers (CRCs) [10], whereas germline SMAD4 mutations are responsible for a subset of patients affected by juvenile polyposis syndrome (JPS; Online Mendelian Inheritance in Man 174900) [4], an autosomal dominant intestinal cancer syndrome. Although the original report showing that SMAD4 germline mutations are responsible for JPS also contained preliminary data indicating that loss of heterozygosity $(\mathrm{LOH})$ of the wild-type allele occurred in a minority of the polyps examined [4], the most convincing evidence for haploinsufficiency at this locus came from the analysis of mouse models for juvenile polyposis. We and others showed that mice carrying targeted Smad4 mutations develop gastrointestinal (GI) polyps with initial retention of the wild-type Smad4 allele; complete functional loss only occurs at later stages of tumor progression within the epithelial compartment $[5,6]$. Nota- bly, loss of a single Smad4 allele in the T-cell compartment and not in the intestinal epithelium resulted in mice characterized by hyperplasia and polyp formation in the GI tract, similar to the animals with constitutive Smad4 mutations [11]. These data indicate that Smad4 haploinsufficiency is likely to play a causative role in GI tumor formation by exerting a 'landscaping' effect from within the microenvironment as originally proposed by Kinzler and Vogelstein [12], and its complete loss of function in the epithelial cells at later tumor stages accompanies progression towards malignancy $[5,6]$. Whether SMAD4 causes polyp formation through haploinsufficiency has been challenged by two studies showing that the majority of tumors from JPS patients carrying germline mutations do show $\mathrm{LOH}$ at the wild-type locus [13,14]. These somatic events occurred both in the epithelial and stromal components of JPS polyps but not in the infiltrating lymphocytes [13].

Here, we have analyzed a cohort of juvenile polyps from patients with germline SMAD4 mutations for the retention of protein expression and confirmed that haploinsufficiency characterizes early stages of polyp formation in a substantial proportion of the cases. Moreover, we attempted the elucidation of the molecular basis of Smad4 haploinsufficiency by studying signal transduction and global gene expression in otherwise normal Smad4+/- cells.

\section{Results \\ SMAD4 haploinsufficiency underlies human juvenile polyposis}

Although haploinsufficiency has been thoroughly characterized in mouse models carrying targeted mutations in the Smad4 gene $[5,6]$, two reports have shown that in the majority of tumors from JPS patients carrying SMAD4 germline mutations $\mathrm{LOH}$ could be detected at the wildtype allele $[13,14]$. To clarify this apparent discordance between mouse and man, and to establish whether SMAD4 behaves as a classical tumor suppressor gene or if, analogous to the Smad4 mouse models, haploinsufficiency underlies early stages of tumor formation in man, we performed SMAD4 immunohistochemistry (IHC) analysis of juvenile polyps from six unrelated JPS patients with known SMAD4 germline mutations (Table 1). Out of 
Table I: Results of the immunohistochemical analysis of intestinal polyps from Juvenile Polyposis syndrome patients carrying established SMAD4 germline mutations.

\begin{tabular}{|c|c|c|c|c|}
\hline & \multicolumn{2}{|l|}{ SMAD4 Germline Mutation } & \multirow[t]{2}{*}{ Epithelial SMAD4 expression } & \multirow[t]{2}{*}{ Stromal SMAD4 expression } \\
\hline & Nucleotide [ref.] & Amino Acid & & \\
\hline \multicolumn{5}{|c|}{ JPS case \#I } \\
\hline polyp A & nt 1042-43, 2 bp del, TTGTTA-TTTA [4] & FS $350 X$ & - & + \\
\hline polyp B & nt I042-43, 2 bp del, TTGTTA-TTTA [4] & FS $350 \mathrm{X}$ & - & - \\
\hline \multicolumn{5}{|c|}{ JPS case \#2 } \\
\hline polyp A & nt 424+I, intron 2 TTGg-TTGa & splice defect & $+/-$ & + \\
\hline \multicolumn{5}{|c|}{ JPS case \#3 } \\
\hline polyp A & nt 1058 TAC-TCC [40] & Tyr353Ser & $+/-$ & + \\
\hline polyp B & nt 1058 TAC-TCC $[40]$ & Tyr353Ser & + & + \\
\hline polyp C & nt 1058 TAC-TCC [40] & Tyr353Ser & + & + \\
\hline \multicolumn{5}{|c|}{ JPS case \#4 } \\
\hline polyp A & nt 533 TㅡA-TㅡA [40] & Ser $178 X$ & $+/-$ & + \\
\hline polyp B & 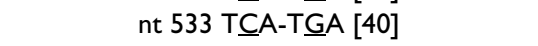 & Serl78X & + & + \\
\hline \multicolumn{5}{|c|}{ JPS case \#5 } \\
\hline polyp A & $\begin{array}{c}\text { nt 687-692, I bp ins, TGGGGGGC- } \\
\text { TGGGGGGGC [4] }\end{array}$ & FS 235X & + & + \\
\hline polyp B & $\begin{array}{c}\text { nt 687-692, I bp ins, TGGGGGGC- } \\
\text { TGGGGGGGC [4] }\end{array}$ & FS 235X & - & - \\
\hline polyp C & $\begin{array}{c}\text { nt 687-692, I bp ins, TGGGGGGC- } \\
\text { TGGGEGGGC [4] }\end{array}$ & FS 235X & $+/-$ & $+/-$ \\
\hline \multicolumn{5}{|c|}{ JPS case \#6 } \\
\hline polyp A & $\begin{array}{c}\text { nt } 1244-47,4 \text { bp del, AGACAGAG-AGAG } \\
{[4]}\end{array}$ & FS 434X & $+/-$ & + \\
\hline polyp B & $\begin{array}{c}\text { nt I244-47, } 4 \text { bp del, AGACAGAG-AGAG } \\
{[4]}\end{array}$ & FS 434X & $+1-$ & + \\
\hline
\end{tabular}

In the last two columns, +, +/-, and - indicate homogeneously positive, patchy, and homogeneously negative nuclear SMAD4 staining, respectively (for examples of the various staining patterns, see Figure I).

13 polyps analyzed, three $(23 \%)$ revealed a homogeneously negative staining of the epithelial tumor cells, thus indicating functional loss of the wild-type SMAD4 allele (Figure $1 \mathrm{~g}$ and $1 \mathrm{~h}$ ). In the remaining cases, SMAD4 expression was either patchy with groups of negative glands scattered among positive ones $(n=6,46 \%$; Figure $1 \mathrm{c}$ to $1 \mathrm{f})$, or showed clear retention of SMAD4 expression $(n=4,31 \%$; Figure $1 \mathrm{a}$ and $1 \mathrm{~b})$. As far as the tumor-associated stroma is concerned, different mesenchymal cell types, for example stromal fibroblasts and infiltrating lymphocytes, showed in almost all cases SMAD4 nuclear reactivity amidst a variable number of negative cells (Figure 1). However, no relationship could be established between the percentage of SMAD4-positive stromal cells and the loss/retention of expression in the adjacent epithelial glands. Also, although the total number of tumors analyzed is admittedly small, no correlation could be found between polyp size and loss/retention of SMAD4 expression.

Overall, the IHC results confirm that in a substantial proportion of the polyps here analyzed from JPS patients with SMAD4 germline mutations, tumor onset does not follow Knudson's two-hit model as SMAD4 expression is retained in either all or in a considerable proportion of the epithelial tumor cells. In these cases, as previously shown in the Smad4+/E6sad mouse model [6], haploinsufficiency is likely to underlie juvenile polyp onset, whereas later stages of tumor progression are accompanied by loss of the wild-type allele.

\section{Smad4 haploinsufficiency results in partial inhibition of TGF- $\beta$ and BMP signaling}

In order to study the transcriptional and signal transduction defects arising from haploinsufficiency at the Smad4 tumor suppressor gene, we employed mouse embryonic stem (ES) cells where a null mutation, namely a single nucleotide deletion in the exon 6 splice acceptor site resulting in an unstable mRNA, is present in the endogenous locus $[6,15]$. The choice of ES cells as a cellular model to study Smad4 haploinsufficiency was made mainly based on observations that different components of the parenchymal and microenvironmental compartments are likely to contribute to polyp initiation and progression to malignancy $[5,6,11]$, as described above. ES cells represent the inner cell mass of the pre-implantation blastocyst and thus precede the differentiation of the three main germ layers. Moreover, the employment of normal rather than neoplastic cells allows bypassing of confounders caused by the altered cellular physiology characteristic of tumor cells and focuses on the primary molecular and cellular consequences of Smad4 haploinsufficiency. The 


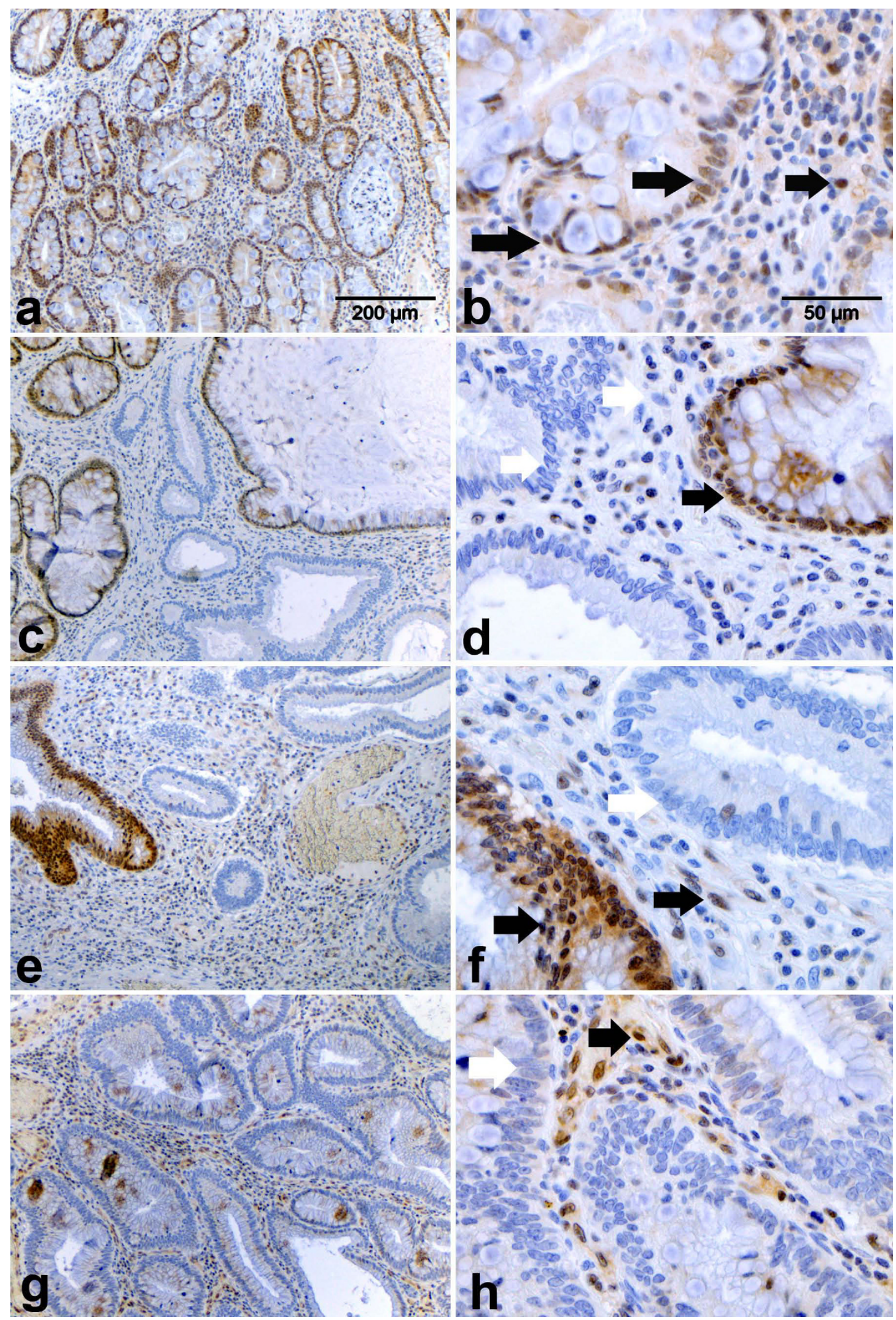

Figure I (see legend on next page) 
Figure I (see previous page)

Immunohistochemical analysis of SMAD4 protein expression in juvenile polyps from patients carrying SMAD4 germline mutations. SMAD4 immunohistochemical analysis of hamartomatous polyps obtained from unrelated Juvenile Polyposis syndrome patients with established SMAD4 germline mutations (see Table I). Images were taken at I0× (a, c, e, g,) and $40 \times$ (b, d, f, h). Filled (black) arrows indicate cells scored as positive, whereas white arrows point to negatives. a-b. Example of a polyp positive for SMAD4 nuclear staining in both the epithelial and stromal compartment (scored as double positive in Table I). c-d. Polyp with heterogeneous SMAD4 expression pattern with patches of positively and negatively staining epithelial glands. Most of the stromal cells appear negative with few exceptions. This tumor was scored as $+/-$ (epithelial) and - (stromal) in Table I. e-f. Example of a juvenile polyp with heterogeneous SMAD4 expression in the epithelial compartment but with a more pronounced positive staining of the stromal tumor microenvironment. This tumor was scored as $+/-($ epithelial) and + (stromal) in Table I. g-h. Example of a juvenile polyp characterized by negative SMAD4 staining throughout the epithelial cells. In the stromal compartment however, several positively staining cells are present. This tumor was scored as - (epithelial) and + (stromal) in Table I.

alternative use of mouse embryonic fibroblasts (MEFs) was made impossible by the early in utero lethality characteristic of the Smad4EGsad/EGsad embryos which precludes MEFs isolation with this genotype [15].

First, we evaluated Smad4 protein expression in wild-type $($ Smad4+/+), heterozygous (Smad4+/E6sad $), \quad$ and homozygous (Smad4 ${ }^{\text {E6sad/EGsad) }}$ ES cells obtained from preimplantation blastocysts of interbred C57Bl6/J Smad4+/ Essad mice. To this aim, two independent hetero- and homozygous ES clones were analyzed by western blot. As shown in Figure 2 (upper panel), Smad4EGsad/E6sad cells did not reveal any protein expression thus confirming the null nature of this mutation, whereas heterozygous ES lines showed a consistent reduction in protein expression when compared with wild-type ES cells, indicative of their haploinsufficiency at the protein level. Moreover, western analysis of intestinal cells from Smad4+/E6sad mice validated the protein haploinsufficiency in adult tissues (Figure 2, lower panel).

To determine whether the observed reduction of Smad4 expression in mouse Smad4+/EGsad ES and intestinal cells impairs TGF- $\beta$ and BMP signaling, we measured the levels of the Smads transcriptional complex by a TGF- $\beta$ reporter assay system [16]. Due to the insufficient expression levels of the type II TGF- $\beta$ receptor (TBRII) in ES cells (data not shown), TGF- $\beta$ reporter assay constructs were co-transfected with an expression vector (pCMV5-TBRII) encoding for the TBRII receptor [17]. A dose-response assay was subsequently performed which indicated that a TGF $\beta 1$ concentration of $50 \mathrm{pmol}$ provides the highest response in the TBRII-transfected cells (data not shown). As depicted in Figure 3A, Smad4E6sad/EGsad ES cells demonstrated a dramatic decrease in TGF- $\beta$ signaling activity when compared with the wild-type controls. Notably, decreased TGF- $\beta$ activity is already apparent in Smad4+l Essad ES clones, characterized by an intermediate yet highly significant $(p<0.001)$ level of luciferase activity between wild-type and homozygous cells. The difference between
Smad4+/+ and Smad4+/E6sad ES cells was not significant when untransfected cells were employed.

Similar results were obtained by analyzing the Smad4mutant ES cells for BMP signaling levels by the BRE-luc reporter assay transfected together with a constitutively active ActR-I receptor (Alk-2) [18]: while Smad4E6sad/E6sad ES cells showed a marked decrease in BMP signaling, haploinsufficient cells revealed an intermediate though significant ( $\mathrm{p}=0.0024$ ) level between wild-type and Smad4deficient ES cells (Figure 3B). Also, rescue of homozygous Smad4EGsad/EGsad ES cells by transfection with a human SMAD4 expression vector [9] fully restored TGF- $\beta$ signaling (Figure 3C).

\section{Smad4 affects genome-wide gene expression in a dosage- dependent fashion}

In order to further characterize the effects of Smad4 haploinsufficiency on gene transcription, expression profiling analysis of total RNA samples from wild-type, Smad4+1 E6sad, and Smad4E6sad/E6sad ES cells was performed using the Affymetrix MOE430 2.0 array. Two independent clones for each genotype were employed for the analysis. Two individual lists of differentially expressed genes were generated by applying a $\mathrm{p}$-value threshold of $p<0.01$ and $p<$ 0.05 for the homo- and heterozygous cells, respectively. Comparison of these two data sets led to the identification of a signature of 79 differentially expressed genes (31 upand 48 down-regulated respectively) common to Smad4 hetero- and homozygous cells, 64 of which (24 up- and 40 down) represent functionally annotated genes (Additional file 1).

Among the differentially expressed entries, a broad spectrum of functional categories is represented: members of known signal transduction pathways (for example, Mdm2, Axin2, Smad7, Zak), growth factors (Fgf5, Fgf8, Igfbp3, Lefty2), immunity related genes (Irgm, Il23a, Cxcl14), and transcription factors (Nrip1, Eomes, Stat3, Cnot6, $T$ brachyury homolog) (Additional file 1). This is 


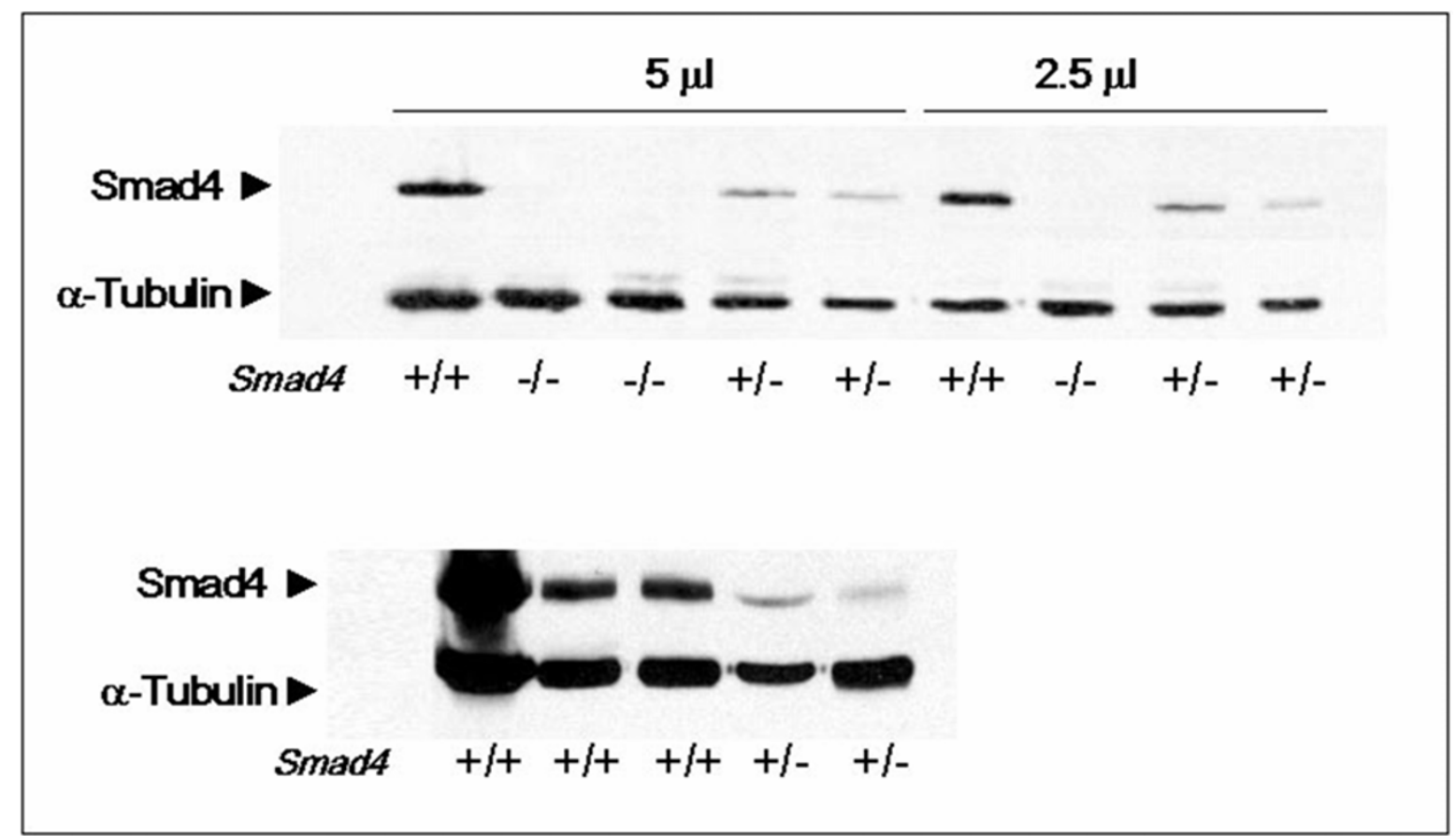

Figure 2

Western analysis of Smad4-mutant embryonic stem cell lines. SMAD4 western blot analysis demonstrates haploinsufficiency in embryonic stem (ES) and adult intestinal cells from Smad4+/E6sad mice. Upper panel: ES cell lysates loaded at two different protein amounts; lower panel: normal intestinal tissue lysates from wild-type and Smad4+/E6sad mice.

also confirmed by Ingenuity Pathway analysis performed on the 64 gene signature (Additional file 2). The presence of Smad4 itself within the list of differentially expressed genes serves as an internal confirmation of the validity of our approach. Notably, when the fold changes levels relative to these 79 genes are plotted in a trend analysis according to the Smad4 genotypes, the Smad4 dosagedependent gradient of transcriptional response becomes apparent (Figure 4).

In order to validate the observed Smad4 dosage-dependent effect on the transcriptional regulation of a subset of target genes, we performed quantitative real-time RT-PCR on laser-capture microdissected (LCM) normal intestinal cells obtained from two wild-type and three Smad4+/EGsad animals. From the list of 64 genes differentially expressed upon Smad4 haploinsufficiency, we selected six known to be expressed in the GI tract and characterized by at least a two-fold change in the ES cell expression profiling data $(\log 2>1)$. As an internal reference standard, we employed the Cryzl (crystallin zeta quinone reductase-like 1) gene, which retains constant expression levels between all ES and adult intestinal cells (data not shown). Similar to the ES cells expression profiling results, both up- (Prkar1b and Tgb1) and down-regulated (Smad7, Irgm, Arts-1 and Igfbp1) genes showed consistent changes in gene expression levels in Smad4+/EGsad normal intestinal cells when compared with intestinal epithelia from wild-type $\left(\right.$ Smad4 $\left.4^{++}\right)$animals (Figure 5), thus validating the microarray expression profiling results.

\section{Discussion}

Over the last few years it has become clear that in a subset of tumor suppressor genes the somatic inactivation or mutation of the wild-type allele (the second hit) does not invariably represent the rate-limiting tumor formation step $[1,3,19]$. It is generally thought that haploinsufficiency may affect normal cell function and homeostasis, possibly in a synergistic manner with other genetic or epigenetic somatic hits at unrelated cancer genes. Both JPS patients and mouse models carrying loss of function mutations at the SMAD4 tumor suppressor gene represent illustrative examples of haploinsufficiency in GI tract tumorigenesis. In partial disagreement with Knudson's two- 
A

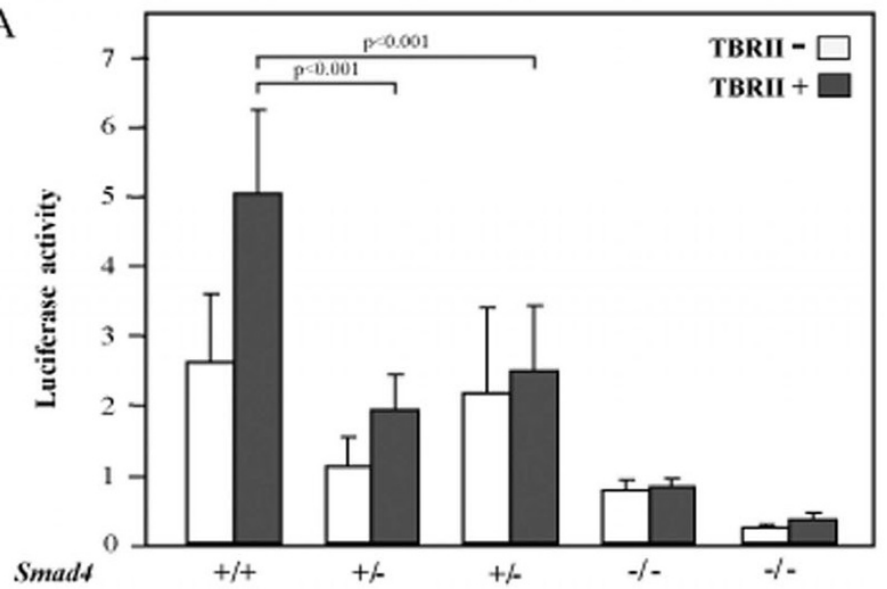

B
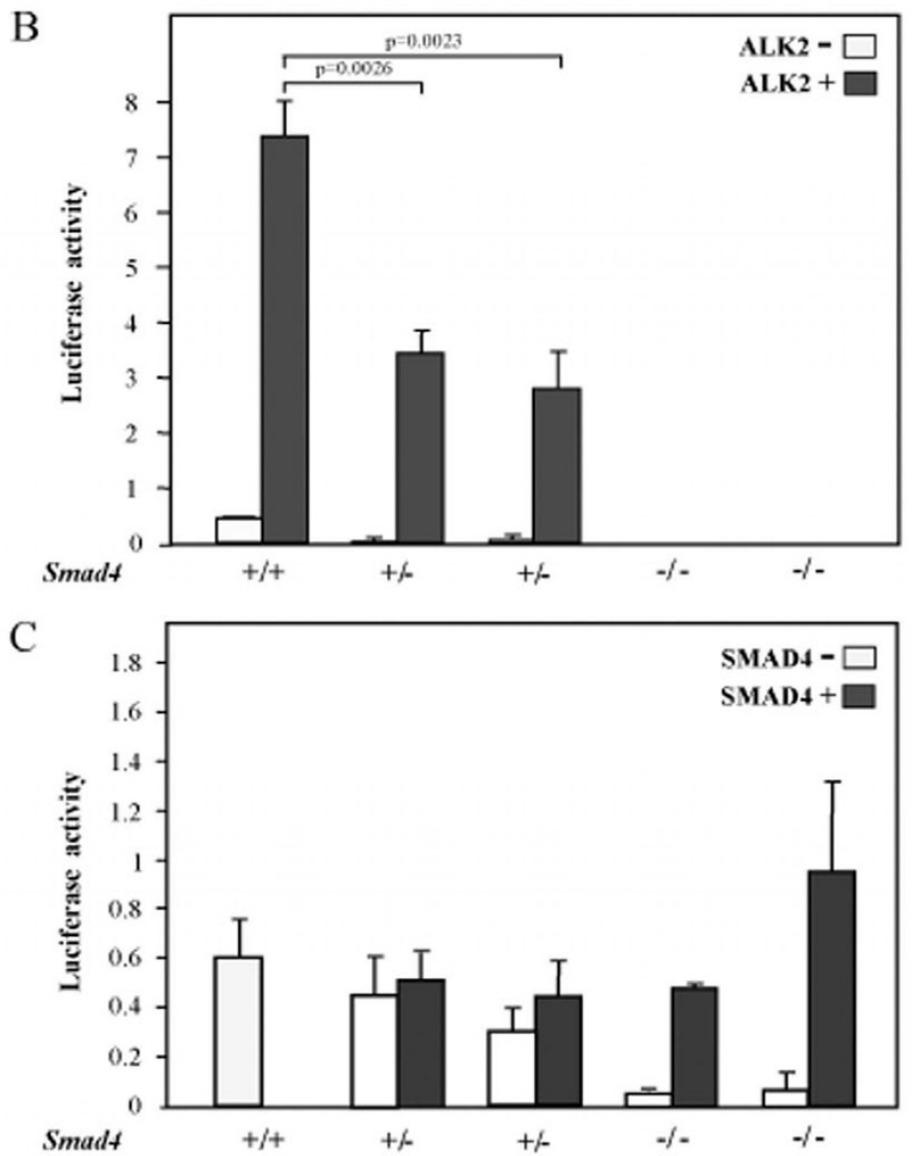

Figure 3

TGF- $\beta$ and BMP reporter assay analysis of Smad4-mutant embryonic stem cell lines. TGF- $\beta$ and BMP reporter assay analysis of Smad4-mutant embryonic stem (ES) cell lines. A. TGF- $\beta$ (CAGA I2-MLP-luciferase) and B. BMP (BRE-luciferase) reporter assays were carried out in wild-type $(+/+)$, Smad4+/Essad (+/-), and Smad4Ebsad/EGsad (-/-) ES cell lines. Normalized CAGA-luc and BRE-luc levels are indicated for each cell line. For each genotype reporter activities are shown for cells transfected (TBRII+ and ALK2+) and non transfected (TBRII- and ALK2-) with the corresponding receptor-expressing vector. In C. the TGF- $\beta$ reporter assays analysis was carried out after transfection with a SMAD4-expressing vector. Each bar represents the average of three independent experiments, and the error bars represent the standard deviation. For the CAGA I2-MLP-luciferase assay, three independent ES wild-type clones have been employed. The WT bar represents the average of the luciferase activities measured in two independent experiments. $p$ values were calculated by the statistical two samples t-test (two tails). 


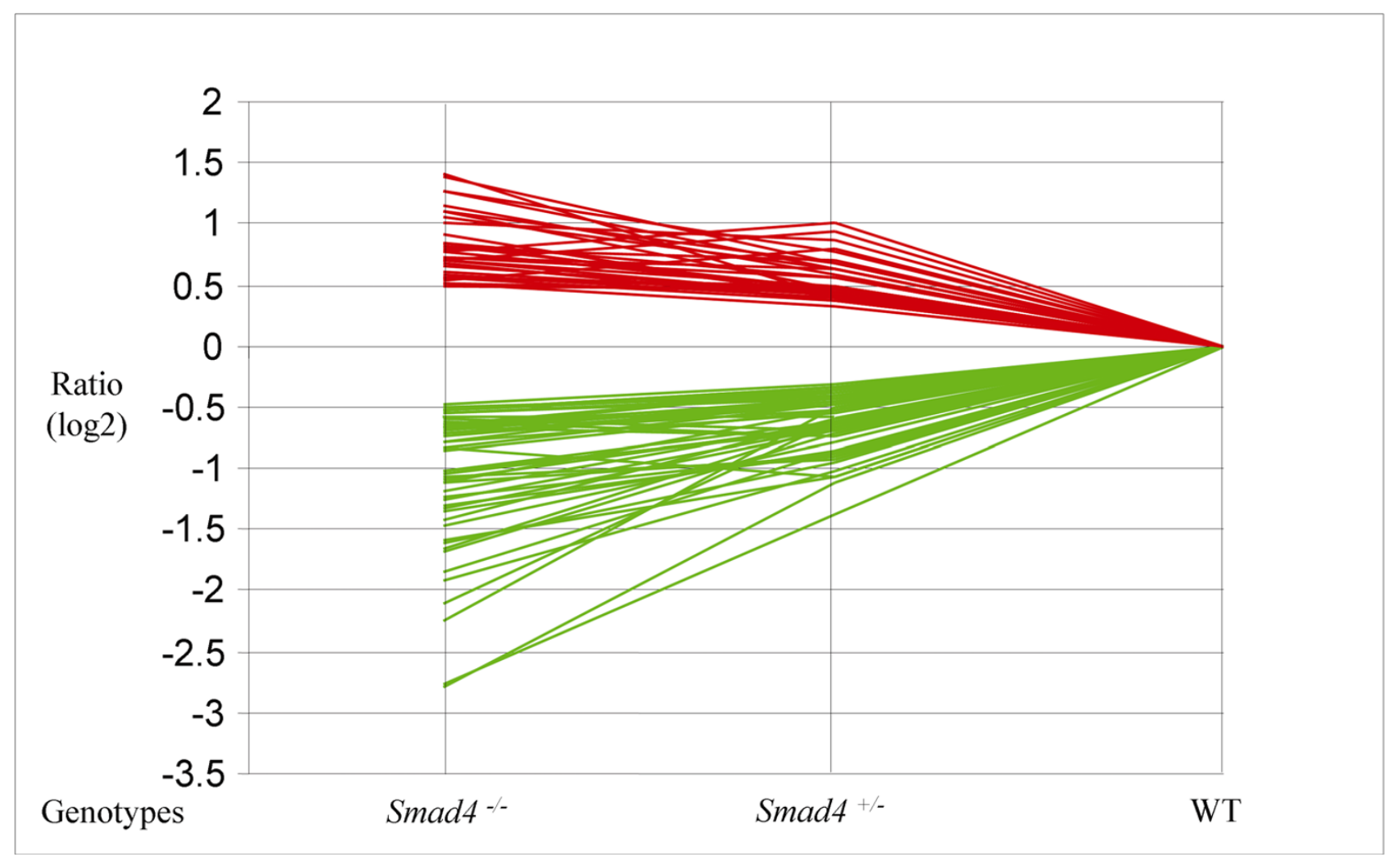

\section{Figure 4}

Trend analysis of Smad4 dosage-dependent transcriptional targets. Trend analysis of the 79 genes found to be differentially expressed in both Smad4+/E6sad and Smad4E6sad/E6sad embryonic stem cells. The fold changes are represented as (log2) ratio values and plotted as red and green lines for up- and down-regulated genes, respectively.

hit model, somatic loss of the wild-type allele is not a ratelimiting event in intestinal polyp formation in Smad4mutant mouse models $[5,6]$. Accordingly, as shown in our study, the majority of polyps from JPS patients carrying SMAD4 germline mutations retain SMAD4 expression both in epithelial tumor cells and in their stromal microenvironment, thus indicating haploinsufficiency. Previously, Howe et al showed that juvenile polyps from SMAD4 mutation carriers reveal loss of the wild-type allele in only $9 \%(1 / 11)$ of their cases [4]. However, the analysis was done by an exon-specific PCR assay unable to detect more subtle somatic hits such as point mutations and epigenetic silencing. Notably, two subsequent and more thorough reports have shown by $\mathrm{LOH}$, fluorescence in situ hybridization, and IHC analysis that loss of the wild-type allele could be detected in the majority of tumors from JPS patients carrying SMAD4 germline muta- tions $[13,14]$. Moreover, SMAD4 loss was observed in both epithelial and some of the stromal cells, which was interpreted by the authors as an indication of the clonal origin of these lesions, and of the fact that SMAD4 represents a classical 'gatekeeper' tumor suppressor rather than a 'landscaper' as originally proposed $[12,13]$. How can the apparent discordance between the present study and the reports by Woodford-Richens and colleagues be solved? From the IHC analysis depicted in Figure 1 it should be clear that a high degree of heterogeneity in SMAD4 expression characterizes juvenile polyps both in the epithelial and mesenchymal compartments. This heterogeneity, when reduced to more quantitative values as in the case of PCR-based LOH analysis of whole tumor specimens comprehensive of both parenchymal and microenvironmental cells, may result in loss of accuracy. Also, differences in interpretation of IHC images may partly underlie this dis- 

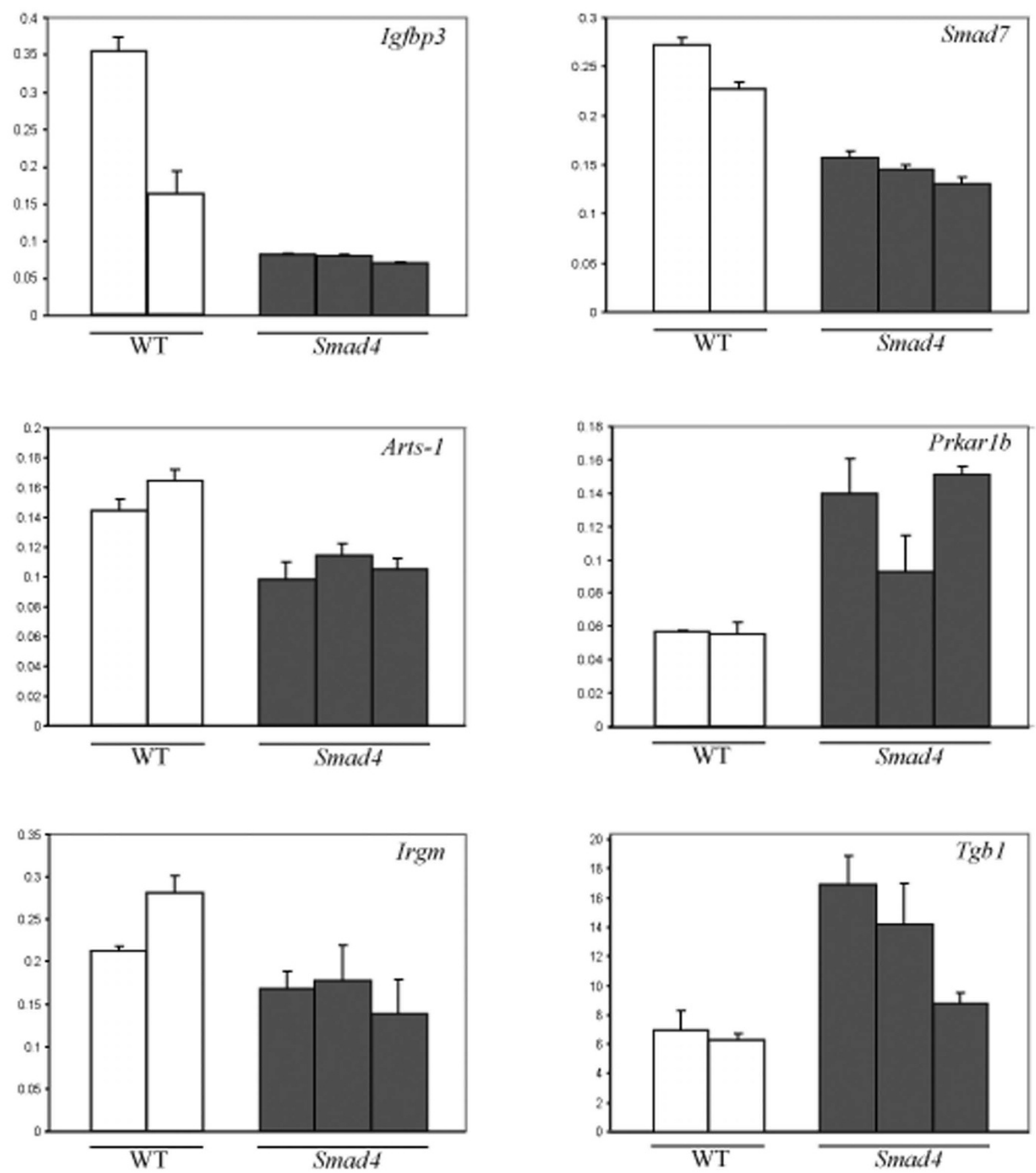

Figure 5

qPCR validation of the expression profiling results. Quantitative PCR analysis of a selection of six genes differentially regulated in Smad4+/E6sad cells. Gene expression was quantified in normal intestinal tissues from two wild-type (WT) and three Smad4+/E6sad (Smad4) animals and is plotted as ratio over the reference gene (see Materials and Methods). Each bar represents the average of three independent experiments. 
crepancy. In their IHC analysis of juvenile polyps from SMAD4-mutant JPS patients [14], Woodford-Richens et al present an example where, similar to observations in our study, a heterogeneous staining pattern is observed with positive epithelial glands amidst negative ones. Last but not least, both the two-hit and haploinsufficiency models appear to hold true for SMAD4-driven tumorigenesis, and this may depend on the molecular nature and pathogenicity of the first hit, namely the germline mutation. As predicted by the 'just right' model for the APC tumor suppressor gene $[20,21]$, the molecular nature of the first hit at a tumor suppressor locus affects the type of secondhit mutation at the wild-type allele. It is plausible to think that while some SMAD4 mutations require functional inactivation of the wild-type allele to trigger tumor formation, others can result in juvenile polyp onset without this otherwise rate-limiting somatic step.

A second important aspect is relative to the role of the SMAD4 in tumor formation either as an epithelial 'gatekeeper' or as a 'landscaper', that is, acting from within the microenvironment to affect epithelial homeostasis [12]. Recently, it was shown that selective loss of Smad4 in the mouse T-cell compartment results in intestinal adenomas reminiscent of JPS polyps [11]. Notably, loss of a single Smad4 allele in T-cells also resulted in hyperplasia and polyp formation in the intestinal epithelial layer, thus indicating that Smad4 haploinsufficiency plays a causative role in GI tumor formation by exerting a 'landscaping' effect from within the stromal compartment [11]. Conversely, our own observation, according to which more advanced lesions of the Smad4+/E6sad mouse model show complete loss of Smad4 expression [6], is indicative of an additional role for the complete loss of Smad4 function in the epithelial compartment at later tumor progression stages. Whether the same holds true for SMAD4-driven juvenile polyp formation in man is still debatable. From our own IHC analysis (Figure 1), it should be evident that the SMAD4 expression pattern in the tumor microenvironment appears rather heterogeneous, with a mixture of positive and negative stromal fibroblasts and infiltrating lymphocytes. However, these results are inconclusive in discriminating between the 'gatekeeper' versus 'landscaper' scenarios. In fact, there is little doubt about the active role played by the tumor microenvironment, especially in the presence of a TGF- $\beta$ signaling defect. Previous reports have shown that loss of function mutations at the TBRII gene can both trigger epithelial tumorigenesis from the stromal layer [22] and underlie malignant transformation when induced in the parenchymal cells of intestinal adenomas initiated by $A p c$ mutations [23]. Similar observations were made for the LKB1 gene (also known as STK11), responsible for Peutz-Jeghers syndrome (PJS; OMIM 175200), an autosomal dominant predisposition to hamartomas (polyps of the GI tract with a pronounced mesenchymal component, very similar to those characteristic of JPS). $L k b 1^{+/-}$mice develop intestinal polyps which often retain the wild type allele [24]. Accordingly, LOH is not an obligate step in polyps from PJS patients with germline LKB1 mutations [25]. Notably, monoallelic loss of murine $L k b 1$ in the smooth muscle compartment results in GI polyps indistinguishable from those observed in mice and in PJS patients with a constitutive mutation, thus confirming the 'landscaping' role of $L k b 1$ haploinsufficiency [26]. Further molecular analysis of these mice revealed that the partial loss of Lkb1 function results in a TGF- $\beta$ signaling defect within the stromal compartment likely to contribute to polyp formation by generating a permissive microenvironment for the malignant transformation of the epithelium [26]. Given the heterogeneous composition of the tumor microenvironment comprising not only stromal fibroblasts but also smooth muscle cells and various cellular types of immune origin, it is plausible to think that haploinsufficiency at members of the TGF- $\beta$ signaling pathways such as SMAD4 and LKB1 affects cell to cell communication in different tissues, thus leading to loss of tissue architecture.

In this study, we have shown that Smad4 haploinsufficiency results in dosage-dependent inhibition of TGF- $\beta$ and BMP signaling, thus affecting epithelial cell proliferation and differentiation both in the stromal and epithelial compartments and likely to underlie juvenile polyp formation in the intestinal tract. Also, expression profiling of Smad4 haploinsufficient cells revealed the existence of a subset of target genes whose expression is specifically regulated by decreased dosages of this tumor suppressor, presumably through TGF- $\beta$ and BMP signaling, as also shown by the differential expression of two known downstream targets of the TGF- $\beta$ pathway, namely Smad7 and Tgfb1. Smad7 is both an inhibitor of TGF- $\beta$ signaling and itself a TGF- $\beta$ downstream target [27]. Hence, Smad7 down-regulation, as observed in the Smad4+/E6sad ES cells, reflects the observed TGF- $\beta$ signaling inhibition. Tgfb1 is an extracellular protein with only poorly characterized functions, though its significant over-expression, as observed in Smad4-mutant ES cell lines, has also been reported in sporadic CRC [28].

Among the genes shown to be differentially up- or downregulated in a Smad4 dosage-dependent fashion, members of other signal transduction pathways were also included (Additional files 1 and 2). Mdm2 up-regulation, as observed in Smad4+/EGsad cells, may favor tumor transformation by inhibiting p53-mediated transactivation [29] and by destabilizing retinoblastoma (RB). The gene encoding for the scaffold protein Axin2 (conductin), down-regulated in Smad4+/EGsad cells, has been previously implicated in canonical Wnt signaling and colorectal pathogenesis [30]. The latter is indicative of cross-talking 
between TGF- $\beta$ and Wnt signal transduction already in haploinsufficiency. Down-regulation of the Igfbp3 gene, encoding for the insulin growth factor binding protein, in Smad4+/EGsad ES and intestinal cells may also represent a relevant early step in tumor formation. Igfbp3 has been described as tumor suppressor gene [31], due to its role in the regulation of cell proliferation and apoptosis, and its differential methylation in a substantial fraction of CRC cases [32].

Overall, Smad4 haploinsufficiency affects both TGF- $\beta$ and BMP signaling together with a broad spectrum of transcriptional targets and cellular functions. Future studies will reveal which of these target genes are preferentially affected in a specific cellular compartment, and the paracrine or cell autonomous effect that they exert on epithelial tumorigenesis.

\section{Conclusion}

In this study we have shown that haploinsufficiency at the SMAD4 tumor suppressor locus underlies polyp formation in a proportion of GI tumors from JPS patients, as previously shown in Smad4-mutant mouse models. Moreover, SMAD4 haploinsufficiency affects both the TGF- $\beta$ and BMP signal transduction pathways together with a broad spectrum of transcriptional targets and cellular functions. These results contribute to our understanding of the cellular and molecular mechanisms underlying intestinal tumorigenesis due to TGF- $\beta$ (and BMP) signaling defects not only in the parenchymal cells but also from within the stromal microenvironment. This is of fundamental but also of clinical relevance as these dosagespecific transcriptional targets may offer novel opportunities in the development of tailor-made therapeutic strategies.

\section{Methods \\ Generation of Smad4-mutant ES cell lines}

Smad4+/E6sad mice, available on the inbred C57Bl/6J background, were inter-bred and the resulting blastocysts harvested at $3.5 \mathrm{dpc}$. Flushed pre-implantation blastocysts were then individually cultured on 96-well dishes coated with MEFs as previously described [33].

All mouse experiments were performed upon approval of the local animal experiment committee (DEC permissions nr. EUR 596, 600, 623 and 730) and according to internationally recognized guidelines (as described by the Code of Practice Dierproeven in het Kankeronderzoek).

\section{Smad4 western analysis}

Equal amounts $(40 \mu \mathrm{g})$ of protein lysates were separated on $12 \%$ SDS polyacrylamide gels, and further subjected to immunoblotting according to standard procedures. Several studies have validated the specificity and sensitivity of the B-8; sc-7966 monoclonal antibody against SMAD4 (Santa Cruz Biotechnology) to detect alterations of protein expression in both mouse and human specimens. The B-8; sc-7966 primary antibody was employed for western analysis at a 1:100 dilution. Peroxidase-conjugated secondary antibodies (Jackson Immunoresearch) were visualized with an enhanced chemiluminescence system.

\section{Reporter assay analysis}

ES cells grown on tissue culture dishes coated by mitotically inactivated primary MEFs were transfected by Lipofectamine 2000 (Life Technologies) with $250 \mathrm{ng}$ of the reporter plasmid ((CAGA)12-MLP-luciferase for the TGF$\beta$ signaling or BRE-luciferase for the BMP signaling), 100 ng of receptor-expressing vector (TGFBRII or, for BMP signaling, a constitutively active form of ALK2) [17,18] (all kindly provided by Professor P ten Dijke), and $5 \mathrm{ng}$ of a Renilla reniformis luciferase-expressing vector. For the rescue experiments, $100 \mathrm{ng}$ of the Smad4-pCMV5 expression vector [9] were transfected together with (CAGA) 12-MLPluciferase and TGFBRII. After 24 hours, ES cells transfected with the (CAGA)12-MLP-luciferase were stimulated with recombinant human TGF- $\beta$ (50 pmol) for 1 hour before measuring luciferase activity with the luminometer Fluoroskan Ascent CF (Labsystems) using the Dual Luciferase Reporter Assay system (Promega). Luciferase activities were calculated as a ratio between the specific luciferasereporter construct and the Renilla luciferase levels, for a total of three different experiments, each carried out in triplicate. For the CAGA12-MLP-luciferase assay, three independent ES wild-type clones were used and the average of their luciferase activities measured in two independent experiments.

\section{Immunohistochemical analysis}

Formalin-fixed, paraffin-embedded intestinal polyps were prepared as $4 \mu \mathrm{m}$ sections and immunostained with the mouse Smad4 B-8; sc-7966 monoclonal antibody directed against Smad4 (Santa Cruz Biotechnology Inc, dilution $1: 100$ ). After antigen retrieval treatment (10 min boiling in Tris-EDTA pH 8.0), endogenous peroxidase was inactivated with $1 \% \mathrm{H}_{2} \mathrm{O}_{2} /$ PBS. A 30 min pre-incubation step in 5\% non-fat dry milk in PBS was followed by incubation with the Smad 4 antibody overnight at $4{ }^{\circ} \mathrm{C}$ in preincubation buffer. Sections were then stained with the Envision HRP-ChemMate kit (DAKO). Smad4 IHC staining was evaluated after brief hematoxylin counterstaining of the slides.

\section{Expression profiling analysis}

Total RNA was labeled using the GeneChip One-Cycle Target Labeling and Control Reagents kit, and hybridized to MOE430 2.0 Affymetrix oligonucleotide arrays, according to the manufacturers' instructions. Raw signal intensities were extracted and summarized from cel-files, 
Table 2: Primers used in real-time RT-PCR analysis

\begin{tabular}{lll}
\hline Gene & Forward Primer & Reverse Primer \\
\hline Cryzll & 5'-AGCTGCTGGCGTCATCCG-3' & 5'-CTGTGGTGGGCTAACTGAATGG-3' \\
Smad4 & 5'-GTGACTGTGGATGGCTATGTGG-3' & 5'-GCAACCTCGCTCTCTCAATCG-3' \\
Arts-I & 5'-GCAGACTTGGACAGATGAAGG-3' & 5'-TGACTTCCACTCTCTGAAATAGC-3' \\
Smad7 & 5'-TGCCTCGGACAGCTCAATTCG-3' & 5'-CCCACACGCCATCCACTTCC-3' \\
Prkarlb & 5'-GCCCGAATCCCTGTCCCTTG-3' & 5'-TGGCTGGCTCATATCACACTCC-3' \\
Irgm & 5'-ACAGGCTCCAGCAGGTTACC-3' & 5'-TTGCCACAGTCTCCTTGATTCC-3' \\
Tgfbl & 5'-CAAACAGGCGTCAGCGTATTCC-3' & 5'-GGCTCTCCTCCTCGGTCTTCC-3' \\
Igfbpl & 5'-CCCAGAGGCGTCCACATCC-3' & 5'-GTCCACACACCAGCAGAAGC-3' \\
\hline
\end{tabular}

followed by normalization using the robust multi-array average expression measure implemented in the Bioconductor package affylmGUI [34]. No filtering was applied to the data. A Bayesian linear regression model was used to detect differentially expressed genes implemented in the Bioconductor package limma [35,36]. All Bioconductor packages were used with R statistical Computing Software v2.2.1 [37].

\section{Quantitative real-time RT-PCR analysis}

LCM of intestinal tissues (approx. 6000 cells) was performed as previously described [38]. PCR analysis was carried out in triplicate in $25 \mu \mathrm{l}$ volumes using $1 \mu \mathrm{l}$ of cDNA and SYBR ${ }^{\circledast}$ Green Dye (Applied Biosystems) on the MyiQ Single-Color Real-Time PCR Detection System (Bio-Rad). Primers sequences are listed below. Standard curves for the target genes and the reference Cryzl1 gene were generated and the normalization and ratio were calculated as described [39], (see Table 2).

\section{Competing interests}

The authors declare that they have no competing interests.

\section{Authors' contributions}

PA was responsible for the immunohistochemistry, reporter assay, western and Q-PCR analyses, and has been involved in drafting the manuscript. CG performed the microarray analysis and the bioinformatic analysis of the resulting data. PF was mainly responsible for the histological processing of the tumor tissues and the SMAD4 immunohistochemistry analysis. MMG contributed to the target validation by quantitative PCR. IdV carried out the TGF- $\beta$ reporter assays. RJS, AR, and LAA contributed the intestinal polyps from JPS patients with SMAD4 germline mutations. RF designed and supervised the study and wrote the final manuscript. All authors have read and approved the final manuscript.

\section{Additional material}

\section{Additional file 1}

Supplementary Table 1. List of 64 functionally annotated genes differentially express in Smad4+/E6sad and Smad4 4 Essad/EGsad ES cell lines. Expression profiling values are expressed as absolute fold change values when compared to Smad $4{ }^{+/+}$ES cells.

Click here for file

[http://www.biomedcentral.com/content/supplementary/17558417-1-2-S1.doc]

\section{Additional file 2}

Supplementary Table 2. Ingenuity Pathway Analysis of the 64 functionally annotated genes differentially expressed (denoted as "focus molecules" in bold) in Smad4 $4^{+/ E}$ Gsad and Smad4 ${ }^{\text {EGsad/EGsad }}$ ES cell lines. The column denoted as "Top Functions" describe the gene ontology groups to which the genes encompassed in a given Ingenuity Network belong. Only the top 4 networks with the most significant scores are included.

Click here for file

[http://www.biomedcentral.com/content/supplementary/17558417-1-2-S2.doc]

\section{Acknowledgements}

Supported was obtained from the Dutch Cancer Society (EMCR 200I2482), the Dutch Research Council (NWO/Vici 016.036.636), the 'Besluit Subsidies Investeringen Kennisinfrastructuur' program of the Dutch Government (BSIK 03038), Dutch Research Council (NWO-Vici), EU FP6 (MCSCs; nr. 037297) EU FP7 (TuMIC; nr. 201662), and the 'Maag Lever Darm Stichting' (MWO 04-2I). The authors are very grateful to Professor $\mathrm{P}$ ten Dijke for his kind donation of the reporter constructs, to $S$ Marttinen for the Finnish JPS patient material, to Dr NH Le for critically reading the manuscript, and to Mr F van der Panne for his help with the artwork.

\section{References}

I. Payne SR, Kemp CJ: Tumor suppressor genetics. Carcinogenesis 2005, 26:203I-2045.

2. Knudson AG Jr: Mutation and cancer: statistical study of retinoblastoma. Proc Natl Acad Sci USA 1971, 68:820-823.

3. Fodde R, Smits R: Cancer biology. A matter of dosage. Science 2002, 298:76I-763.

4. Howe JR, Roth S, Ringold JC, Summers RW, Jarvinen HJ, Sistonen P, Tomlinson IP, Houlston RS, Bevan S, Mitros FA, Stone EM, Aaltonen LA: Mutations in the SMAD4/DPC4 gene in juvenile polyposis. Science 1998, 280: 1086-1088.

5. Xu X, Brodie SG, Yang X, Im YH, Parks WT, Chen L, Zhou YX, Weinstein M, Kim SJ, Deng CX: Haploid loss of the tumor suppressor Smad4/Dpc4 initiates gastric polyposis and cancer in mice. Oncogene 2000, 19:1868-1874. 
6. Alberici P, Jagmohan-Changur S, De Pater E, Valk M Van Der, Smits $\mathrm{R}$, Hohenstein P, Fodde R: Smad4 haploinsufficiency in mouse models for intestinal cancer. Oncogene 2006, 25: |84I-|85|.

7. Heldin $\mathrm{CH}$, Miyazono $\mathrm{K}$, ten Dijke P: TGF-beta signalling from cell membrane to nucleus through SMAD proteins. Nature 1997, 390:465-47|.

8. Duff EK, Clarke AR: Smad4 (DPC4) - a potent tumour suppressor? BrJ Cancer 1998, 78:1615-1619.

9. Nakao A, Imamura T, Souchelnytskyi S, Kawabata M, Ishisaki A, Oeda E, Tamaki K, Hanai J, Heldin CH, Miyazono K, ten Dijke P: TGF-beta receptor-mediated signalling through Smad2, Smad3 and Smad4. Embo J 1997, 16:5353-5362.

10. Salovaara R, Roth S, Loukola A, Launonen V, Sistonen P, Avizienyte E, Kristo $P$, Jarvinen $H$, Souchelnytskyi S, Sarlomo-Rikala M, Aaltonen LA: Frequent loss of SMAD4/DPC4 protein in colorectal cancers. Gut 2002, $51: 56-59$.

II. Kim BG, Li C, Qiao W, Mamura M, Kasprzak B, Anver M, Wolfraim L, Hong S, Mushinski E, Potter M, Kim SJ, Fu XY, Deng C, Letterio J]: Smad4 signalling in $T$ cells is required for suppression of gastrointestinal cancer. Nature 2006, 44I:1015-1019.

12. Kinzler KW, Vogelstein B: Landscaping the cancer terrain. Science 1998, 280: 1036-1037.

13. Woodford-Richens K, Williamson J, Bevan S, Young J, Leggett B, Frayling I, Thway Y, Hodgson S, Kim JC, Iwama T, Novelli M, Sheer D, Poulsom R, Wright N, Houlston R, Tomlinson I: Allelic loss at SMAD4 in polyps from juvenile polyposis patients and use of fluorescence in situ hybridization to demonstrate clonal origin of the epithelium. Cancer Res 2000, 60:2477-2482.

14. Woodford-Richens KL, Rowan AJ, Poulsom R, Bevan S, Salovaara R, Aaltonen LA, Houlston RS, Wright NA, Tomlinson IP: Comprehensive analysis of SMAD4 mutations and protein expression in juvenile polyposis: evidence for a distinct genetic pathway and polyp morphology in SMAD4 mutation carriers. $\mathrm{Am}$ Pathol 200I, 159:1293-1300.

15. Hohenstein $\mathrm{P}$, Molenaar L, Elsinga J, Morreau H, Klift $\mathrm{H}$ van der, Struijk A, Jagmohan-Changur S, Smits R, van Kranen H, van Ommen GJ, Cornelisse C, Devilee P, Fodde R: Serrated adenomas and mixed polyposis caused by a splice acceptor deletion in the mouse Smad4 gene. Genes Chromosomes Cancer 2003, 36:273-282.

16. Dennler S, Itoh S, Vivien D, ten Dijke P, Huet S, Gauthier JM: Direct binding of Smad3 and Smad4 to critical TGF beta-inducible elements in the promoter of human plasminogen activator inhibitor-type I gene. Embo J |998, I7:309|-3100.

17. Wrana JL, Attisano L, Carcamo J, Zentella A, Doody J, Laiho M, Wang XF, Massague J: TGF beta signals through a heteromeric protein kinase receptor complex. Cell 1992, 71:1003-10I4.

18. Korchynskyi $\mathrm{O}$, ten Dijke $\mathrm{P}$ : Identification and functional characterization of distinct critically important bone morphogenetic protein-specific response elements in the IdI promoter. J Biol Chem 2002, 277:4883-4891.

19. Santarosa M, Ashworth A: Haploinsufficiency for tumour suppressor genes: when you don't need to go all the way. Biochim Biophys Acta 2004, 1654:105-122.

20. Lamlum H, llyas M, Rowan A, Clark S, Johnson V, Bell J, Frayling I, Efstathiou J, Pack K, Payne S, Roylance R, Gorman P, Sheer D, Neale K, Phillips R, Talbot I, Bodmer W, Tomlinson I: The type of somatic mutation at APC in familial adenomatous polyposis is determined by the site of the germline mutation: a new facet to Knudson's 'two-hit' hypothesis. Nat Med 1999, 5: I07|- 1075.

21. Albuquerque $C$, Breukel C, Luijt R van der, Fidalgo P, Lage P, Slors FJ, Leitao CN, Fodde R, Smits R: The 'just-right' signaling model: APC somatic mutations are selected based on a specific level of activation of the beta-catenin signaling cascade. Hum Mol Genet 2002, II:1549-1560.

22. Bhowmick NA, Chytil A, Plieth D, Gorska AE, Dumont N, Shappell S, Washington MK, Neilson EG, Moses HL: TGF-beta signaling in fibroblasts modulates the oncogenic potential of adjacent epithelia. Science 2004, 303:848-851.

23. Munoz NM, Upton M, Rojas A, Washington MK, Lin L, Chytil A, Sozmen EG, Madison BB, Pozzi A, Moon RT, Moses HL, Grady WM Transforming growth factor beta receptor type II inactivation induces the malignant transformation of intestinal neoplasms initiated by Apc mutation. Cancer Res 2006, 66:9837-9844

24. Bardeesy N, Sinha M, Hezel AF, Signoretti S, Hathaway NA, Sharpless NE, Loda M, Carrasco DR, DePinho RA: Loss of the Lkb I tumour suppressor provokes intestinal polyposis but resistance to transformation. Nature 2002, 419:162-167.

25. Hernan I, Roig I, Martin B, Gamundi MJ, Martinez-Gimeno M, Carballo $M$ : De novo germline mutation in the serine-threonine kinase STK I I/LKB I gene associated with Peutz-Jeghers syndrome. Clin Genet 2004, 66:58-62.

26. Katajisto P, Vaahtomeri K, Ekman N, Ventela E, Ristimaki A, Bardeesy N, Feil R, DePinho RA, Makela TP: LKB I signaling in mesenchymal cells required for suppression of gastrointestinal polyposis. Nat Genet 2008, 40:455-459.

27. Nakao A, Afrakhte M, Moren A, Nakayama T, Christian JL, Heuchel $\mathrm{R}$, Itoh S, Kawabata M, Heldin NE, Heldin CH, ten Dijke P: Identification of Smad7, a TGFbeta-inducible antagonist of TGFbeta signalling. Nature 1997, 389:631-635.

28. Buckhaults P, Rago C, St Croix B, Romans KE, Saha S, Zhang L, Vogelstein B, Kinzler KW: Secreted and cell surface genes expressed in benign and malignant colorectal tumors. Cancer Res 2001 , 61:6996-700I.

29. Sdek P, Ying H, Chang DL, Qiu W, Zheng H, Touitou R, Allday MJ, Xiao ZX: MDM2 promotes proteasome-dependent ubiquitinindependent degradation of retinoblastoma protein. Mol Cell 2005, 20:699-708.

30. Liu W, Dong X, Mai M, Seelan RS, Taniguchi K, Krishnadath KK, Halling KC, Cunningham JM, Qian C, Christensen E, Roche PC, Smith DI, Thibodeau SN: Mutations in AXIN2 cause colorectal cancer with defective mismatch repair by activating beta-catenin/ TCF signalling. Nat Genet 2000, 26: I46-I47.

31. Xi Y, Nakajima G, Hamil T, Fodstad O, Riker A, Ju J: Association of insulin-like growth factor binding protein-3 expression with melanoma progression. Mol Cancer Ther 2006, 5:3078-3084.

32. Tomii K, Tsukuda K, Toyooka S, Dote H, Hanafusa T, Asano H, Naitou M, Doihara H, Kisimoto T, Katayama H, Pass HI, Date H, Shimizu $\mathrm{N}$ : Aberrant promoter methylation of insulin-like growth factor binding protein-3 gene in human cancers. Int J Cancer 2007, I 20:566-573.

33. Helms AW, Matise MP, Joyner AL: Establishing new ES lines. In Gene Targeting, A Practical Approach Edited by: Joyner AL. Oxford: Oxford University Press; 2000: I29-131.

34. Wettenhall JM, Smyth GK: limmaGUI: a graphical user interface for linear modeling of microarray data. Bioinformatics 2004, 20:3705-3706.

35. Smyth GK: Limma: linear models for microarray data. In Bioinformatics and Computational Biology Solutions using $R$ and Bioconductor Edited by: Gentleman VCR, Dudoit S, Irizarry R, Huber W. New York: Springer; 2005:397-420.

36. Smyth GK: Linear models and empirical bayes methods for assessing differential expression in microarray experiments. Stat Appl Genet Mol Biol 2004, 3:Article 3.

37. $R$ Development Core Team: A language and environment for statistical computing. Vienna: R Foundation for Statistical Computing; 2005.

38. Alberici P, de Pater E, Cardoso J, Bevelander M, Molenaar L, Jonkers J. Fodde R: Aneuploidy arises at early stages of apc-driven intestinal tumorigenesis and pinpoints conserved chromosomal loci of allelic imbalance between mouse and human. Am J Pathol 2007, 170:377-387.

39. Pfaffl MW: A new mathematical model for relative quantification in real-time RT-PCR. Nucleic Acids Res 200I, 29:e45

40. Roth $S$, Sistonen $P$, Salovaara R, Hemminki $A$, Loukola $A$, Johansson M, Avizienyte E, Cleary KA, Lynch P, Amos Cl, Kristo P, Mecklin JP, Kellokumpu I, Jarvinen $\mathrm{H}$, Aaltonen LA: SMAD genes in juvenile polyposis. Genes Chromosomes Cancer 1999, 26:54-6I. 